\title{
IMPROVING THE PERFORMANCE OF NIRS-BASED BRAIN-COMPUTER INTERFACES IN THE PRESENCE OF BACKGROUND AUDITORY DISTRACTIONS
}

\author{
Tiago H. Falk, K. Paton, S. Power, and Tom Chau \\ Bloorview Research Institute/Bloorview Kids Rehab \\ Institute of Biomaterials and Biomedical Engineering \\ University of Toronto, Toronto, Canada
}

\begin{abstract}
In this paper, the effects of auditory distractions on the performance of brain-computer interfaces (BCI) based on nearinfrared spectroscopy (NIRS) are investigated. Experiments show that NIRS-BCI specificity decreases by an average $19 \%$ when operated in the presence of continuous background noise (relative to operation in silence) and by $13 \%$ when operated in the presence of startle noises. To improve BCI performance in noisy environments, a simple yet effective startle noise compensation strategy is proposed. Acoustic environmental conditions are tracked in realtime and false BCI activations that occur within seconds of detected startle noises are suppressed. Experiments show NIRS-BCI systems equipped with the proposed compensation system attaining performances in noisy conditions comparable to those attained in silent conditions.
\end{abstract}

Index Terms - Brain-computer interface, near-infrared spectroscopy, hidden Markov model, auditory distraction.

\section{INTRODUCTION}

Brain-computer interfaces (BCI) have emerged as promising access solutions for individuals with severe and multiple disabilities. BCIs can harness electric, magnetic, or metabolic brain signals to allow individuals to interact with their surrounding environment and to control external devices such as a computer, a wheelchair, or a neuroprosthesis. Recently, near-infrared spectroscopy (NIRS) has been used as a reliable method of non-invasively measuring cortical hemodynamic responses for BCI design [1].

NIRS is an analysis technique used to map the hemodynamic response in the brain that occurs as a result of mental activation. Near-infrared (650 nm - $950 \mathrm{~nm}$ wavelengths) electromagnetic radiation is directed incident into the scalp allowing the majority of the radiation (light) to pass into the cortex to a depth of 1-3 cm with little interference [1]. When voluntary brain activation occurs, metabolic demands and increased blood flow result in changes to the regional concentrations of oxygenated and deoxygenated hemoglobin, thus altering the optical properties of the brain [2]. The fraction of light absorbed versus the fraction reflected is mostly dependent on the concentrations of such hemoglobins, as they are the two primary absorbers of near-infrared light in the brain tissue. By measuring the intensity of the reflected light, previous research has used NIRS technologies to assess hemodynamic responses in the motor cortex during motor imagery activity [1] and in the prefrontal cortex during music imagery [3] or mental calculation tasks [4].

To improve NIRS-based BCI performance, significant efforts have been placed on the detection and removal of physiological noise (e.g., motion artifacts, respiration and heart rate) from the recorded spectroscopy signals [2]. Little research, however, has been conducted to investigate the effects of auditory distractions on NIRS-BCI performance. Previous studies, however, have suggested that the prefrontal cortex is involved in the processing of auditory information, particularly of distracting auditory stimuli $[5,6]$. As a consequence, it is expected that the performance of NIRS-BCI technologies based on prefrontal cortex activation (e.g., via music imagery) will be compromised in noisy environments. In order to develop robust technologies that can be taken beyond experimental scenarios and into a user's typical surroundings, the effect of such environmental factors must be ascertained.

In this paper, the first steps towards automated compensation of environmental noise artifacts on NIRS-BCI performance are taken. The developed BCI makes use of hidden Markov models (HMM) to characterize NIRS signals of individuals at rest. Control signals are developed based on automatic detection of music imagery events via an HMM log-likelihood measure. Experiments show that BCI performance decreases significantly under constant background and startle noise (e.g., door slamming, phone ringing) conditions. A simple yet effective startle noise compensation strategy is proposed and used to suppress erroneous BCI activations caused by involuntary cortical hemodynamic responses. Performance levels close to those attained in silent conditions are attained once noise compensation is in place.

The remainder of this paper is organized as follows. Section 2 describes the NIRS-BCI, Section 3 presents the experimental results and conclusions are drawn in Section 4. 


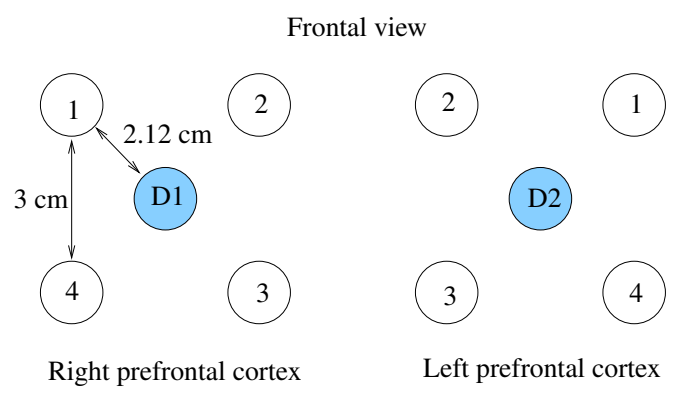

Fig. 1. Positioning of source pairs (circles) and detectors (shaded circles). Each source pair represents one $\lambda=690 \mathrm{~nm}$ source and one $\lambda=830 \mathrm{~nm}$ source.

\section{NIRS-BASED BRAIN-COMPUTER INTERFACE}

In this section, the multiple components used in the development of the NIRS-BCI are described.

\subsection{NIRS Signal Measurement}

Hemodynamic responses were recorded using an Imagent Function Brain Imaging System from ISS Inc. Two photomultiplier tube detectors were employed along with sixteen light sources - eight at $690 \mathrm{~nm}$ and eight at $830 \mathrm{~nm}$. The sources delivered $110 \mathrm{MHz}$-modulated light to the forehead via $400 \mu \mathrm{m}$-diameter optical fibres, and the detectors received the returning light via $3 \mathrm{~mm}$-diameter optical fibres. Light returned to the detectors was demodulated at a cross-correlation frequency $(\mathrm{CCF})$ of $5 \mathrm{kHz}$. To avoid cross-signal contamination, the light sources were cyclically switched such that no two sources were on simultaneously. The effective sampling rate was $31.25 \mathrm{~Hz}$ and three output data components were collected: AC (relative amplitude at the CCF), DC (relative amplitude at $0 \mathrm{~Hz}$ ), and phase.

Positioning of four source pairs around one detector on each side of the participant's forehead allowed both the right and left prefrontal cortices to be probed, as depicted by Fig. 1. Areas probed included the frontopolar cortex, the superior portion of the orbitofrontal cortex, and medial sections of the dorsolateral prefrontal cortex. Emitters in position 3 on each side were located roughly at the left and right prefrontal cortices (FP1 and FP2 in the 10-20 system). On each side, the four source pairs were positioned $2.12 \mathrm{~cm}$ away from the detector, a distance that has been shown to suffice for cortical hemodynamic probing [7].

\subsection{Pre-Processing and Feature Extraction}

The raw signals (AC and DC) were wavelet-denoised to suppress physiological noise due, primarily, to cardiac signals $(0.5-2 \mathrm{~Hz})$, respiration $(0.2-0.4 \mathrm{~Hz})$ and the Mayer wave (approximately $0.1 \mathrm{~Hz}$ ) [2]. Here, three wavelet (12-level Daubechie) filters are explored and the filtered signals com- prise of the reconstruction of the approximation wavelet coefficients and either the last three, four or five detail coefficients. AC and DC signals are denoted as $x_{p, i}^{\lambda}(\gamma, d)$, where the subscript $i$ indexes the source position $(i=1, \ldots 4)$, subscript $p$ indicates the left or right hemisphere of the forehead by $L$ or $R$, respectively, the superscript $\lambda$ indicates the wavelength ( $\lambda=690,830 \mathrm{~nm})$, the signal type (AC or DC) is indicated by the parameter $\gamma$ and parameter $d$ indicates the 'detail' of the filtered signal $(d=3,4,5)$.

Additionally, estimated changes in oxygenated $\left(\mathrm{HbO}_{2}\right)$ and deoxygenated $(\mathrm{HHb})$ hemoglobin concentrations were computed using the modified Beer-Lambert law [1] and the raw $\mathrm{AC}$ and DC signals. The notation $c_{p, i}^{h}(d)$ is used to denote oxygenated ( $h=\mathrm{HbO}_{2}$ ) or deoxygenated hemoglobin $(h=$ $H H b$ ) concentrations, and parameters $p=L, R, i=1, \ldots 4$, and $d=3,4,5$ are used as before. The like-wavelength raw signals (AC and DC) and like-chromophore concentration signals were averaged over the four measurement positions on each side (see Fig. 1) and served as feature vectors to train user-dependent hidden Markov models.

\subsection{User-Centred HMM BCI}

Figure 2 depicts the block diagram of the proposed HMMbased BCI system. Hidden Markov models with Gaussian mixture model output probability distributions are used to model normative hemodynamic responses of individuals at rest (termed "baseline"). Deviations from such models are indicative of music imagery events which, in turn, are used for BCI control; deviations are measured via a normalized log-likelihood measure computed with the forward-backward procedure described in [8]. Startle noise detection is employed to improve performance in noisy conditions.

During training, hidden Markov model parameters such as state transition probabilities, initial state probabilities, and output distribution parameters are computed using the expectation-maximization algorithm summarized in [8]. To allow for a user-centred approach, different HMM configurations are explored per subject. Pilot experiments were conducted to investigate reliable values for the number of HMM states $Q$ and the number of Gaussian mixture components $M$ per state; full covariance Gaussian components were used. Three parameter combinations were shown to strike a good balance between complexity and performance, namely: $Q=4$ and $M=1$ or $Q=2$ and $M=1$ or 2 .

For each participant, HMMs were trained for different combinations of $\mathrm{AC}, \mathrm{DC}$, or concentration features using the training baseline signals. More specifically, the fourdimensional feature vectors include:

$$
\begin{aligned}
\vec{u}_{D C, d}= & {\left[\bar{x}_{L}^{690}(D C, d), \bar{x}_{L}^{830}(D C, d), \bar{x}_{R}^{690}(D C, d), \bar{x}_{R}^{830}(D C, d)\right], } \\
\vec{u}_{A C, d}= & {\left[\bar{x}_{L}^{690}(A C, d), \bar{x}_{L}^{830}(A C, d), \bar{x}_{R}^{690}(A C, d), \bar{x}_{R}^{830}(A C, d)\right], } \\
& \vec{u}_{c o n c, d}=\left[\bar{c}_{L}^{H b}(d), \bar{c}_{L}^{H b O}(d), \bar{c}_{R}^{H b}(d), \bar{c}_{R}^{H b O}(d)\right],
\end{aligned}
$$

where $d$ indexes 3-, 4-, or 5-detail wavelet filters. 


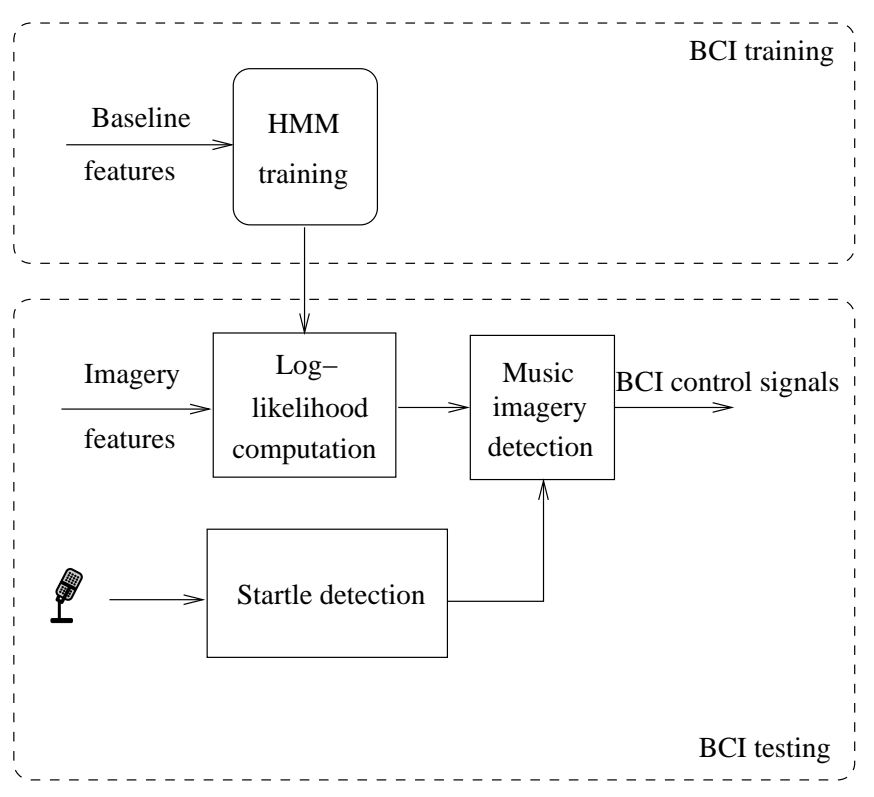

Fig. 2. Block diagram of the proposed NIRS-BCI with startle compensation achieved via environment sniffing.

During testing, log-likelihood values are computed for consecutive and overlapping frames of the imagery test data. Here, frame lengths of $l=1,3,5,7,10$, and 15 seconds are investigated with 0.5 -second overlap. Normalization is performed based on the length of the window. Window lengths of different sizes are investigated as reaction times may differ between subjects due to various external factors such as mental alertness, reaction time, and familiarity with the procedure. Higher log-likelihood values suggest hemodynamic responses akin to those observed during rest. Lower loglikelihood values, in turn, indicate responses different from rest, i.e, music imagery events.

For music imagery detection, a simple classification approach is employed; more specifically, changes from positive to negative slope in the log likelihood function are used. In order to remove possible artifacts due to e.g., head movement, music imagery events were only detected if a decrease in the $\log$ likelihood function persisted for at least four seconds. Additionally, in order to compensate for false activations due to involuntary hemodynamic responses resultant from auditory distractions, a startle noise detection algorithm is employed based on the "environment sniffing" paradigm [9]. Once startle noises are detected, false BCI activations are suppressed. Such compensation strategy will be shown to improve BCI performance under noisy conditions (see Section 3.3).

\section{EXPERIMENTAL RESULTS}

In this section, the collected data is described and experimental results are reported.

\subsection{Data Collection}

Twelve subjects performed each a total of 8 trials over two separate days. Half the trials were performed in silence and the other half with auditory distractions. Of the trials performed in silence, two consisted of alternating periods of music imagery and rest (termed "imagery" trials and used as test signals), and two of the individual at rest with no music imagery (termed "baseline" trials and used as training signals). Of the trials performed under noisy conditions, half consisted of imagery trials with continuous babble noise played in the background and the other half of imagery trials with startle noises (sneeze, dog barking, glass breaking, door slamming, and a cough) played randomly in the background. Before each session began, each participant chose at least two songs of the same emotional valence (i.e. 'happy' or 'sad') which he/she felt would elicit an intense emotional response; the selected songs were then used for the music imagery portions of the trials.

The 220-second imagery trials each consisted of eleven 20 -second intervals alternating between rest (six intervals) and music imagery (five intervals), for both the silence and noisy conditions. For the rest intervals, participants were instructed to clear their minds as much as possible, and to focus on their breathing. For the imagery intervals, instructions were to silently sing the pre-selected songs to themselves in their head. An experimenter cued the subjects to transition from rest to imagery and vice versa with a light tap on the arm. The two baseline trials consisted of 120-second rest intervals without music imagery.

\subsection{Performance Metrics}

BCI sensitivity and specificity are used as performance metrics and are given by

$$
\begin{aligned}
\text { Sensitivity } & =\frac{\mathrm{TP}}{\mathrm{TP}+\mathrm{FN}} \times 100 \%, \\
\text { Specificity } & =\frac{\mathrm{TN}}{\mathrm{TN}+\mathrm{FP}} \times 100 \%,
\end{aligned}
$$

where TP and TN refer to true positives and negatives, respectively; FP and FN refer to false positives (erroneous BCI activation) and negatives (undetected music imagery).

\subsection{Experimental Results}

To allow for a user-centred design, optimal combinations of filter detail $d$, HMM parameters $(Q$ and $M)$, and loglikelihood window sizes $l$ are obtained on a per-participant basis. Under silent conditions, the proposed HMM-based BCI attained an average sensitivity of $0.83,0.85$, and 0.87 for $\mathrm{AC}, \mathrm{DC}$, and concentration data, respectively; standard deviations (std) were $0.12,0.13$, and 0.14 . To quantify the effects of false positives caused by auditory distractions, Table 1 further reports the specificity values obtained for each of the 
Table 1. Per-participant specificity values obtained with the HMM-based NIRS-BCI under silent, noisy, and startlecompensated conditions for AC, DC, and concentration data.

\begin{tabular}{|c|c|c|c|c|c|c|c|c|c|c|c|c|}
\hline \multirow[b]{2}{*}{ subject } & \multicolumn{3}{|c|}{ Silence } & \multicolumn{3}{|c|}{ Background } & \multicolumn{3}{|c|}{ Startle } & \multicolumn{3}{|c|}{ Startle Compensation } \\
\hline & $\mathrm{AC}$ & DC & Conc. & $\mathrm{AC}$ & DC & Conc. & $\mathrm{AC}$ & $\mathrm{DC}$ & Conc. & $\mathrm{AC}$ & $\mathrm{DC}$ & Conc. \\
\hline 1 & 1 & 0.92 & 1 & 0.92 & 0.75 & 0.73 & 0.92 & 0.92 & 0.92 & 0.92 & 0.92 & 0.92 \\
\hline 2 & 0.67 & 0.58 & 1 & 0.58 & 0.67 & 0.50 & 0.58 & 0.5 & 0.5 & 0.67 & 0.67 & 0.67 \\
\hline 3 & 0.75 & 0.83 & 0.92 & 0.58 & 0.58 & 0.92 & 0.75 & 0.75 & 0.75 & 0.75 & 0.75 & 0.75 \\
\hline 4 & 0.92 & 1 & 0.7 & 0.83 & 0.92 & 0.86 & 0.92 & 0.91 & 0.91 & 1 & 0.91 & 0.91 \\
\hline 5 & 0.75 & 0.75 & 0.83 & 0.67 & 0.50 & 0.50 & 0.6 & 0.58 & 0.58 & 0.6 & 0.58 & 0.58 \\
\hline 6 & 0.83 & 0.58 & 0.83 & 0.67 & 0.67 & 0.78 & 0.83 & 0.57 & 0.83 & 0.83 & 0.83 & 0.83 \\
\hline 7 & 0.92 & 0.67 & 0.86 & 0.67 & 0.42 & 1.00 & 0.92 & 0.75 & 0.75 & 1 & 0.75 & 1 \\
\hline 8 & 1 & 0.92 & 0.92 & 0.58 & 0.67 & 0.75 & 0.75 & 0.75 & 0.75 & 0.75 & 0.75 & 0.75 \\
\hline 9 & 0.67 & 1 & 0.64 & 0.50 & 0.50 & 0.75 & 0.67 & 0.5 & 0.67 & 0.83 & 0.83 & 0.83 \\
\hline 10 & 1 & 0.92 & 0.75 & 0.67 & 0.67 & 0.92 & 0.83 & 0.58 & 0.58 & 0.83 & 0.58 & 0.58 \\
\hline 11 & 0.92 & 0.83 & 1 & 0.58 & 0.50 & 0.83 & 1 & 0.67 & 0.67 & 1 & 1 & 1 \\
\hline 12 & 0.83 & 0.75 & 1 & 0.83 & 0.42 & 1.00 & 0.83 & 0.83 & 0.83 & 0.92 & 0.83 & 0.83 \\
\hline mean & 0.86 & 0.81 & 0.87 & 0.67 & 0.60 & 0.79 & 0.80 & 0.69 & 0.73 & 0.84 & 0.78 & 0.80 \\
\hline std & 0.12 & 0.15 & 0.13 & 0.13 & 0.15 & 0.17 & 0.13 & 0.15 & 0.13 & 0.13 & 0.13 & 0.14 \\
\hline
\end{tabular}

twelve participants under silent and noisy (background and startle) conditions. For brevity, sensitivity values for noise conditions are not reported as they were not significantly affected. As observed, auditory distractions significantly degrade BCI performance. Raw AC and DC data are more severely affected by constant background noise relative to startle noises; the converse is true for concentration data. Once startle compensation is in place, performance values close to those obtained in silence are attained.

\section{CONCLUSIONS}

The effects of auditory distractions on NIRS-BCI performance are explored. Experimental results suggest that constant background noise degrades average BCI performance by approximately $19 \%$ whereas startle noises degrade performance by an average $13 \%$. Once startle compensation is in place, performance is only degraded by approximately $4.5 \%$. Ongoing investigations include the development of compensation strategies for constant background noise.

\section{REFERENCES}

[1] R. Sitaram et al., "Temporal classification of multichannel near-infrared spectroscopy signals of motor imagery for developing a brain-computer interface," NeuroImage, vol. 34, pp. 1416-1427, 2007.

[2] F. Matthews, B. A. Pearlmutter, T. E. Ward, C. Soraghan, and C. Markham, "Hemodynamics for Brain-Computer Interfaces," IEEE Signal Processing Magazine, vol. 25, no. 1, pp. 87-94, January 2008.
[3] A. Blood and R. Zatorre, "Intensely pleasurable responses to music correlate with activity in brain regions implicated in reward and emotion," Proc. National Academy Sciences, vol. 98, pp. 818-823, 2001.

[4] A. Villringer and B. Chance, "Non-invasive optical spectroscopy and imaging of human brain function," Trends in Neurosciences, vol. 20, no. 10, pp. 431-433, October 1997.

[5] A. Engelien, W. Huber, D. Silbersweig, E. Stern, C. Frith, W. Doring, A. Thron, and R. Frackowiak, "The neural correlates of 'deaf-hearing' in man: Conscious sensory awareness enabled by attentional modulation," Brain, vol. 123 , no. 3, p. 532, 2000.

[6] M. Boly et al., "Auditory Processing in Severely Brain Injured Patients Differences Between the Minimally Conscious State and the Persistent Vegetative State," Archives Neurology, vol. 61, no. 2, pp. 233-238, 2004.

[7] I. Sase, H. Eda, A. Seiyama, H. C. Tanabea, A. Takatsuke, and T. Yanagida, "Multi-channel optical mapping: Investigation of depth information," Proceedings of SPIE, vol. 4250, 2001.

[8] L. R. Rabiner, "A Tutorial on Hidden Markov Models and Selected Applications in Speech Recognition," Proc. IEEE, vol. 77, no. 2, pp. 257-286, Feb. 1989.

[9] M. Akbacak and J. Hansen, "Environmental sniffing: noise knowledge estimation for robust speech systems," IEEE Trans. Audio, Speech, and Lang. Proc., vol. 15, no. 2, pp. 465-477, 2007. 